\title{
Targeting genes and signaling pathways of transcriptional suppressor ZHX2 in hepatocellular carcinoma: a Chromatin Immunoprecipitation-sequencing (ChIP-seq) investigation
}

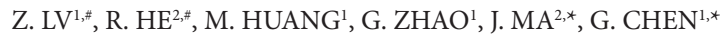 \\ ${ }^{1}$ Department of Pathology, First Affiliated Hospital of Guangxi Medical University, Nanning, Guangxi Zhuang Autonomous Region 530021, \\ China; ${ }^{2}$ Department of Medical Oncology, First Affiliated Hospital of Guangxi Medical University, Nanning, Guangxi Zhuang Autonomous \\ Region 530021, China
}

${ }^{*}$ Correspondence: chengang@gxmu.edu.cn, chengang@gxmu.edu.cn

"Contributed equally to this work.

Received August 6, 2018 / Accepted December 11, 2018

\begin{abstract}
Hepatocellular carcinoma (HCC) is one of the most common malignancies in the world. The unclear underlying molecular mechanisms could provide important theoretical basis for prevention and control of HCC. In this study, chromatin immunoprecipitation-sequencing (ChIP-seq) was performed to analyze the binding sites of zinc fingers and homeoboxes 2 (ZHX2) and its genome-wide target genes, and bioinformatics was used to analyze their gene transcription regulation network. Immunohistochemistry detected the ZHX2 expression in HCC, and its association with clinicopathological characteristics of HCC. Results of RT-PCR and western blot showed that the ZHX2 expression in HepG2 cells was obviously lower compared to normal liver cells. ZHX2 could be amplified in ChIP products and ChIP-seq revealed there were 232 genes binding in promoter regions. GO function analysis revealed these genes were mainly associated with biological processes (BP), cellular components (CC), and molecular functions (MF). In addition, PTEN was found enriched in certain biological functions in BP analysis. Then, four pathways of these genes based on Kyoto Encyclopedia of Genes and Genomes (KEGG) were found $\mathrm{p}<0.05$. Last immunohistochemistry analysis showed the rates of ZHX2 and PTEN expression in paracancerous tissues were both significantly higher than in the HCC tissues $(\mathrm{p}=0.042 ; \mathrm{p}<0.001)$, with negative correlations with AFP values $(r=-0.246, p=0.040 ; r=-0.263, p=0.028)$. Further, PTEN expression was positively correlated with the differentiation level in HCC tissues $(r=0.267, \mathrm{p}=0.025)$. Spearman correlation analysis revealed that the expression profiles of ZHX2 and PTEN were positively correlated in HCC tissues $(r=0.258, p=0.031)$. This study is the first to use ChIP-seq technology to analyze the specific regulatory mechanisms of the transcription suppressor ZHX2 in the context of HCC at the genome level.
\end{abstract}

Key words: hepatocellular carcinoma, HCC, transcription repressor, ZHX2, ChIP-Seq

Hepatocellular carcinoma (HCC) is one of the most common malignancies in the world. On average, approximately 316000 people die of HCC in China every year; therefore, it poses a great threat to human health [1-6]. To provide an important theoretical basis for the prevention and control of HCC, increasing numbers of scholars are investigating the molecular mechanisms underlying the development and progression of HCC [7-11]. ZHX2 is a member of the ZHX protein family and is a transcriptional suppressor localized to cell nuclei. The target genes negatively regulated by ZHX2 include alpha fetoprotein (AFP) and glypican-3 (GPC3) [12, 13]. It has been shown that $\mathrm{ZHX} 2$ regulates the cell cycle proteins cyclin E and cyclin A to play a tumor suppressor role in HCC [14]. A recent study showed that ZHX2 can inhibit the expression of the MDR1 gene, which exerts a drug resistance function in HCC treatment [15]. These studies have all indicated that the transcription factor ZHX2 might interact with many genes in HCC and participate in the development and progression of HCC. In addition, many ZHX2 target genes are still unknown. To target the regulatory mechanisms of the transcriptional suppressor ZHX2 in HCC, this study first performed chromatin immunoprecipitation-sequencing (ChIP-seq) to analyze the binding sites between ZHX2 and its genome-wide target genes and used bioinformatics to analyze their gene transcription regulation network. Next, ZHX2 expression in HCC was detected using immunohis- 
tochemistry, and its association with the clinicopathological characteristics and significance of the disease were analyzed to provide a basis for the pathogenic mechanism of HCC.

\section{Materials and methods}

Materials. Three liver cancer cells HepG2, SMMC7721, Bel7402 and one normal liver cell LO2 were purchased from the Shanghai Cell Bank of the Chinese Academy of Sciences and was passaged and cryopreserved in our laboratory. The ChIP-seq reagent kit was purchased from Merck Millipore (Germany). The rabbit anti-human ZHX2 antibody was purchased from Gene Tex (USA). The PTEN antibody (ready-to-use) was purchased from Beijing Zhongshanjinqiao Biological Technology Co., Ltd. And the anti-mouse and anti-rabbit universal secondary antibodies were purchased from Shanghai Long Island Biotec (China).

Patient samples. A total of 70 cases of stored paraffin blocks from HCC tissues and the corresponding normal paracancerous tissues after surgery were collected from the Department of Pathology in the First Affiliated Hospital of Guangxi Medical University between December 2013 and January 2015. There were 60 male patients and 10 female patients, ranging in age from $20-77$ years, with a mean age of 49.27 years. The tumor histological grading was performed based on the Edmondson standard [16]. No patients received radiotherapy and chemotherapy before surgery, and complete clinical and pathological data, including gender, age, tumor diameter, tumor differentiation, the value of serum $\mathrm{HBsAg}$ and AFP were collected. The study was approved by the Ethics Committee of the First Affiliated Hospital of Guangxi Medical of University. And informed consents were obtained from all patients.

RT-PCR. RNA were purified from four cell lines after lysis using TRIzol Reagent kit. First strand cDNA was synthesized on the base of HiScript ${ }^{\bullet} 1$ st Strand cDNA Synthesis Kit. The resulting cDNAs were used as templates for PCR reactions. The primers used were listed in Table 1. Standard PCR conditions consisted of initial denaturation at $95^{\circ} \mathrm{C}$ for $5 \mathrm{~min}$, followed by 35 cycles at $95^{\circ} \mathrm{C}(45 \mathrm{~s}), 58^{\circ} \mathrm{C}(45 \mathrm{~s})$ and $72^{\circ} \mathrm{C}$ $(60 \mathrm{~s})$ and final elongation at $72^{\circ} \mathrm{C}(10 \mathrm{~min})$. The products were visualized by agarose gel electrophoresis.

Western blot. HepG2 cells were lysed by protein lysate. After cell fragments and lysates were centrifuged, the supernatant was removed to SDS buffer solution and boiled for $5 \mathrm{~min}$; the proteins were isolated by SDS-PAGE and electroblotted onto a nitrocellulose membrane. After blocked with

Table 1. Primer sequences of ZHX2 and GAPDH

\begin{tabular}{ll}
\hline Primer & Sequence \\
\hline ZHX2 Forward primer & GGTAGCGACGAGAACGAG \\
ZHX2 Reverse primer & AGGACTTTGGCACTATGAAC \\
GAPDH Forward primer & TGAGTACGTCGTGGAGTCCA \\
GAPDH Reverse primer & CAAAGTTGTCATGGATGACC \\
\hline
\end{tabular}

non-fat milk, ZHX2 antibody and $\beta$-actin were added for incubation overnight. The next day, membrane was washed by TBST, and horseradish peroxidase was added to mark secondary antibody incubating for 1 hour. ECL luminescence solution was added after washing, and protein bands were visualized by chemiluminescence.

Chromatin immunoprecipitation (ChIP) assay. (1) The culture medium of the HepG2 cells was supplemented with $37 \%$ formaldehyde for fixation. The cross-linking was terminated by adding glycine. The cells were washed twice with pre-cooled phosphate-buffered saline (PBS), resuspended in a mixture of PBS and protease inhibitors, and centrifuged for five min. The supernatant was discarded. (2) Prepared lysis buffer was added to resuspend and lyse the collected cells. The cells were sonicated for 10 min using a sonicator set at the middle range ("Mid" (M)) in ice-cold $\mathrm{H}_{2} \mathrm{O}$, and a $25 \mu \mathrm{l}$ sample was directly cross-linked and purified. (3) The sonicated specimen was divided into three samples. One sample $(25 \mu \mathrm{l})$ was used as the input and was stored at $4{ }^{\circ} \mathrm{C}$. One sample $(250 \mu \mathrm{l})$ was used for the experiments. The experimental tube was filled with $555 \mu$ l of Dilution Buffer containing protease inhibitors, and $50 \mu \mathrm{l}$ of magnetic bead-coupled anti-mouse IgG was added; the solution was mixed for $30 \mathrm{~min}$ at $4^{\circ} \mathrm{C}$. The magnetic beads were absorbed for two min at $4^{\circ} \mathrm{C}$ using a magnetic separation rack. The supernatant was collected and aliquoted into two copies ( $425 \mu \mathrm{l}$ each) and labelled with IP and neg of the corresponding cells; the original magnetic bead was removed; ZHX2 antibody was added to IP tube mixed, with neg tube spied for night at $4{ }^{\circ} \mathrm{C} ; 50 \mu \mathrm{l}$ of magnetic bead was added to IP tube and neg tube, and mixed for $1 \mathrm{~h}$ at $4{ }^{\circ} \mathrm{C}$. The magnetic beads were absorbed for two min at $4^{\circ} \mathrm{C}$ using a magnetic separation rack, and the supernatant was discarded. The magnetic beads were sequentially washed with low-salt, high-salt, and $\mathrm{LiCl}$ wash buffers. The magnetic beads were then washed with TE wash buffer twice; the sample was mixed for five min at $4^{\circ} \mathrm{C}$ each time. After the washes were complete, the magnetic beads were absorbed at $4^{\circ} \mathrm{C}$ for two min using a magnetic separation rack, and the supernatant was discarded. (4) Samples were eluted using CHIP elution buffer, then mixture phenol, chloroform and isoamyl alcohol was added to each reaction tube vibrating for 15 seconds. After samples were centrifuged, upper aqueous phase were transferred to new $\mathrm{EP}$ tubes; $\mathrm{NaCl}$ with glycogen and anhydrous ethanol were added to EP tubes, then EP tubes were placed at $-80^{\circ} \mathrm{C}$ for at least 30 minutes; After precipitation of DNA by centrifuging, the precipitation were washed with $70 \%$ ethanol solution. Then precipitation were dried at room temperature; Tris- $\mathrm{HCl}$ in $\mathrm{pH} 8.0$ dissolved the precipitate; Finally, DNA concentration was determined.

Validation of ChIP products using quantitative polymerase chain reaction (qPCR). Promoter primer design: The primer used was CHIP anti-ZHX2. The three sample tubes included the negative IgG group, the $\mathrm{ZHX} 2$ IP group, and the input group. $\mathrm{H}_{2} \mathrm{O}$, Taq PCR Master Mix, template, and primers were sequentially added. The PCR 
system mixture consisted of $1 \mu \mathrm{l}$ of cDNA template, $0.5 \mu \mathrm{l}$ of upstream primer, $0.5 \mu$ l of downstream primer, $10 \mu$ l of LightCycler 480 SYBR Green Master, and $8 \mu \mathrm{l}$ of dd $\mathrm{H}_{2} \mathrm{O}$. PCR cycle conditions were as follows: $94^{\circ} \mathrm{C}$ pre-denaturation for five min, 35 cycles of $95^{\circ} \mathrm{C}$ for $45 \mathrm{~s}, 58^{\circ} \mathrm{C}$ for $45 \mathrm{~s}$, and $95^{\circ} \mathrm{C}$ for $5 \mathrm{~s}, 72^{\circ} \mathrm{C}$ for $60 \mathrm{~s}$, and $40^{\circ} \mathrm{C}$ for $30 \mathrm{~s}$. For the qPCR analysis, the percentage of positive target DNA fragments among the ChIP input was calculated.

Construction of the ChIP-seq library. The DNA quality in the ChIP samples was controlled. The termini of the DNA fragments were repaired, and an A base was added to the 3 ' end. The sequencing apparatus was connected, and the machine was operated according to the instructions. The DNA fragment size was selected at 300-700 bp. After cluster amplification, the samples were sequenced. Data collection and analysis were then performed. MACS software (specific for ChIP-seq analysis) was used for the analysis and detection of peak values obtained from ChIP-seq data. The potential regulatory sites of the $\mathrm{ZHX} 2$ transcription factor were obtained using MACS analyses.

GO and pathway analysis. The Gene Ontology database (gene ontology, GO) (http://www.geneontology.org/) was used for the classification and functional analysis of genes with ZHX2 binding sites [17-22]. Genes with ZHX2 binding sites were used for pathway enrichment analyses based on the Kyoto Encyclopedia of Genes and Genomes (KEGG, http://www.genome.jp) [20, 23-29]. A p $<0.05$ was set as the threshold value of significant gene enrichment.

Immunohistochemistry (Envision two-step method). (1) Paraffin sections were deparaffinised, and antigen retrieval was performed using the EDTA high-pressure thermal method. Sections were incubated with $3 \% \mathrm{H}_{2} \mathrm{O}_{2}$ at $37^{\circ} \mathrm{C}$ for $10 \mathrm{~min}$ to eliminate endogenous peroxidase activities. Sections were stained with ZHX2 antibody (dilution $1: 100$ ) and incubated at $37^{\circ} \mathrm{C}$ for $1 \mathrm{~h}$. After they were washed with PBS, sections were stained with the secondary antibody and incubated at $37^{\circ} \mathrm{C}$ for $30 \mathrm{~min}$. After diaminobenzidine (DAB) development, sections were washed with PBS, counter-stained with haematoxylin, dehydrated, cleared, and mounted with neutral balsam. The negative control used PBS to replace the primary antibody. Normal lymph node sections

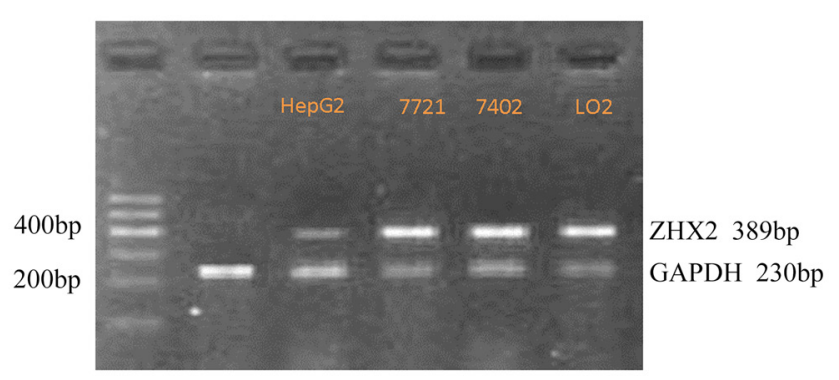

Figure 1. ZHX2 expression in three liver cancer cell lines and one normal hepatic cells. were used as the positive control. (2) Determination of ZHX2 results: According to the darkness of positive cell staining, the results were classified into four levels: (1) no staining $(0$ point), light yellow (1 point), yellow-brown ( 2 points), and brown (3 points). According to the percentage of positive cells, the results were classified into five levels: the percentage of positive cells $<10 \%$ ( 0 points), $10-25 \%$ ( 1 point), $26-50 \%$ ( 2 points), $51-75 \%$ (3 points), and $>75 \%$ ( 4 points) [30] .The sums of the scores of these two items were classified into two levels: $0-3$ points belonged to the negative group, and 4-7 points belonged to the positive group[14]. Determination of PTEN results: positive cells $\leq 5 \%$ obtained 0 points, $6-25 \%$ obtained 1 point, $26-50 \%$ obtained 2 points, $51-75 \%$ obtained 3 points, and $>75 \%$ obtained 4 points; positive intensity showing yellow was 1 point, yellow-brown was 2 points, and brown was 3 points. The score of the cell positive rate was multiplied with the score of the staining intensity [31], with scores of $0-4$ points belonging to the negative group and scores of 5-12 points belonging to the positive group.

Data analyses. All results were statistically analyzed using SPSS 17.0 statistical software. The associations between ZHX2 expression and the clinicopathological characteristics were analyzed using the $\chi^{2}$ test or Fisher's exact probability test. A $p<0.05$ indicated statistical significance.

\section{Results}

Expression of ZHX2 in liver cancer cells compared with normal liver cell. The results of RT-PCR showed ZHX2 mRNA could amplify in four cell lines. The expression of ZHX2 mRNA in HepG2 cells was obviously lower compared with normal liver cells (Figure 1). The protein level of ZHX2 by western blot was corresponding to the result of PCR, lower in HepG2 cells than in LO2 cells (Figure 2).

Validation of $\mathrm{ZHX} 2 \mathrm{ChIP}$ products in HepG2 cells using qPCR. Input and ZHX2 ChIP products were used as templates for amplification. The results showed that $\mathrm{ZHX} 2$ could be amplified in ChIP products with the addition of the ZHX2 antibody in HepG2 cells (Suppl. Figure 1).

Identification of $\mathrm{ZHX} 2$ binding sites. Linkers, repeated copies, contaminated sequences, and low-quality

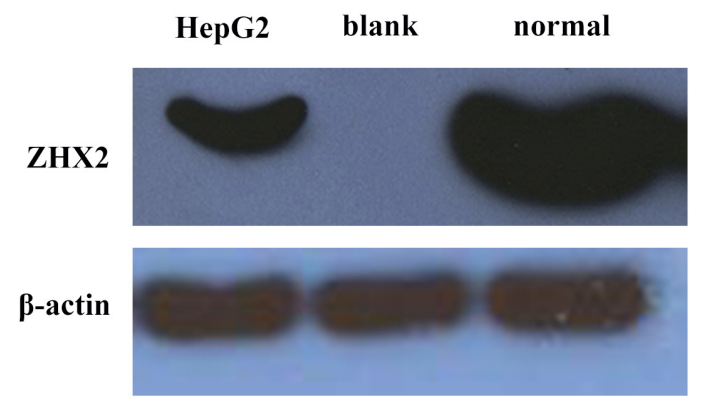

Figure 2. The level of ZHX2 protein expression in HepG2 cells and normal hepatic cells. 
sequences were removed from the raw ChIP-seq data. A total of $31,051,731$ reads were obtained, and the results were compared to the human genome (UCSC hg19) using BOWTIE software to obtain approximately $21,086,492$ reads. Next, MACS v2 software was used to discover 3,093 potential binding sites $(\mathrm{p}<0.05)$.

Distribution of genes with ZHX2 binding sites. The sample peaks obtaining from ChIP-seq reflected the distribution of ZHX2 binding sites in the genome. The newest

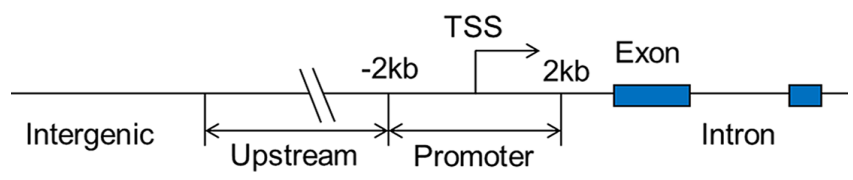

Figure 3. Genomic distribution of the five types of peaks identified by ChIP-seq analysis.

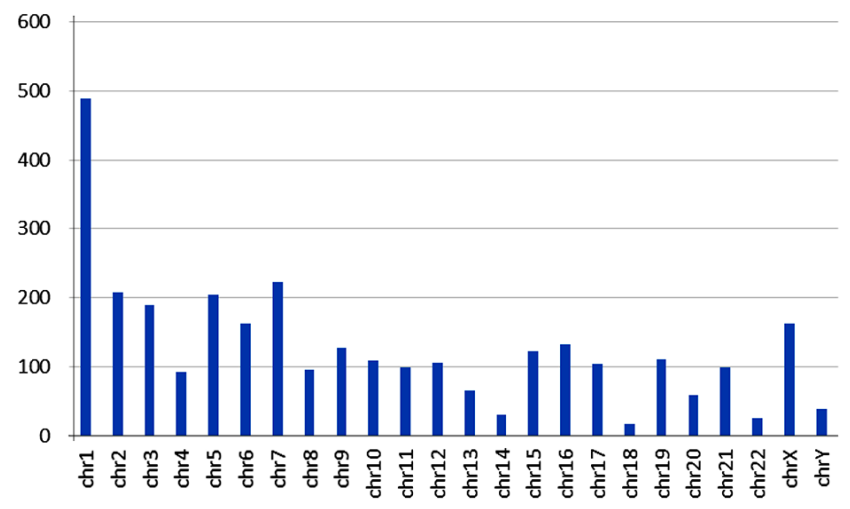

Figure 4. Distribution of ZHX2 binding sites in the functional elements of the genome. The sites were mainly distributed in intergenic regions, followed by introns, upstream regions of promoters, and promoter regions.

Table 2. Localisation of some genes in ZHX2 binding peaks in the genome-wide range of HepG2 cells

\begin{tabular}{lcccc}
\hline Gene & $\begin{array}{c}\text { Fold } \\
\text { enrichment }\end{array}$ & $\begin{array}{c}\text {-LOG10 } \\
\text { (q value) }\end{array}$ & $\begin{array}{c}\text { Peak } \\
\text { classification }\end{array}$ & $\begin{array}{c}\text { Peak } \\
\text { name }\end{array}$ \\
\hline HFM1 & 16.765 & 151.787 & intron & peak_70 \\
ECT2 & 3.964 & 6.482 & exon & peak_1206 \\
PTEN & 3.052 & 4.095 & promoter & peak_324 \\
CMAS & 4.121 & 2.679 & upstream & peak_439 \\
TP53TG3 & 3.681 & 2.822 & intergenic & peak_664 \\
\hline
\end{tabular}

Table 3. Examples of genes bound to the ZHX2 promoter region

\begin{tabular}{lcccc}
\hline Gene & Chr & Name & Peak to TSS & $\begin{array}{c}\text {-LOG10 } \\
\text { (p-value) }\end{array}$ \\
\hline HBB & chr11 & peak 372 & 812 & 6.75035 \\
PC & chr11 & peak 394 & -1504 & 5.55275 \\
MYOC & chr1 & peak 193 & -1888 & 4.70085 \\
PTEN & chr10 & peak 324 & -1726 & 4.0956 \\
CD14 & chr5 & peak 1425 & 1217 & 4.1539 \\
\hline
\end{tabular}

RefSeq database in the University of California Santa Cruz (UCSC) Genome Browser was used for reference. According to their locations, the peak regions were divided into transcription start sites (TSSs), promoter peaks, upstream peaks, intron peaks, and exon peaks. In addition, intergenic peaks indicated peaks that were not localized to the above four peak regions (Figure 3 ). There were approximately 1,981 ZHX2 binding peaks in the genome of the HepG2 HCC cell line. Among these binding sites, approximately 59.32\% were localized in intergenic regions, $20.97 \%$ were in introns, $12.78 \%$ were in the upstream regions of TSSs, $5.76 \%$ were in promoters, and $1.16 \%$ were in exons (Table 2). Analysis of the locations between ZHX2 binding sites and TSSs showed that the majority of binding sites were distributed within 1,000$5,000 \mathrm{bp}$ of TSSs (Figure 4). Comparisons of the chromosome locations of potential ZHX2 binding sites showed that these sites were mainly localized on chromosomes $1,2,3,5$, and 7 , followed by chromosomes $6,9, \mathrm{X}, 15$, and 16 . The Y chromosome had the fewest binding sites (Suppl. Figure 2).

Analysis of ZHX2 binding sites in promoter regions. Analysis of 1981 ZHX2 binding peaks within the range of $2 \mathrm{~kb}$ from a TSS, i.e., analysis of binding sites in promoter regions, revealed that there were 232 genes with ZHX2 binding sites in promoter regions (Table 3 ).

Functional classification of ZHX2-binding genes. Classification and functional analysis of target genes with ZHX2 binding sites were performed using the GO database. GO analysis on genes with ZHX2 binding sites in their promoter regions revealed that 61 genes were enriched in Biological Process (BP), 64 genes were enriched in Cellular Component (CC), and 61 genes were enriched in Molecular Function (MF). According to the significance (p-value) of enrichment, the top five GO items are shown in the following tables (Table 4). Analysis of functions of these genes revealed that they were mainly associated with biological processes (such as biological regulation, metabolic process, and molecular regulation functions), cellular components (such as blood microparticle and pericentric heterochromatin), and molecular functions (such as GTPase activity and activation of other enzymes). In addition, the results of BP analysis showed that PTEN was enriched in certain biological functions, such as negative regulation of cell-matrix adhesion, intracellular connective tissue, and adhesion connective tissues.

KEGG pathway enrichment analyses of ZHX2-regulated genes. To further study the influences of ZHX2 in the development of HCC, the enrichment conditions of differentially expressed genes in the KEGG pathway level were investigated. Four KEGG pathways had $\mathrm{p}<0.05$ (Table 5).

Differential ZHX2 expression between HCC tissues and paracancerous tissues. The ZHX2 protein was positively localized in cell nuclei, with yellow-brown- or brown-colored staining in cell nuclei (Figures 5A, B). The ZHX2 positive expression rates were $57.1 \%$ (40/70) in paracancerous tissues and $40.0 \%$ (28/70) in HCC tissues. The rate of ZHX2 expression in paracancerous tissues was significantly higher than 
Table 4. Partial results of GO enrichment analysis of target genes.

\begin{tabular}{llll}
\hline & \multicolumn{1}{c}{ Term } & \multicolumn{1}{c}{ Ontology } & \multicolumn{1}{c}{ Genes } \\
\hline GO:0043547 & positive regulation of GTPase activity & Biological process & TBC1D3G; TBC1D3K; TBC1D3E; TBC1D3I; TBC1D3B etc. \\
GO:0043087 & regulation of GTPase activity & Biological process & TBC1D3G; TBC1D3K; TBC1D3E; TBC1D3I; TBC1D3B etc. \\
GO:0051345 & positive regulation of hydrolase activity & Biological process & TBC1D3G; TBC1D3K; TBC1D3E; TBC1D3I; TBC1D3B etc. \\
GO:0043085 & positive regulation of catalytic activity & Biological process & GRIN2B; PTEN; TBC1D3G; TBC1D3K; TBC1D3E etc. \\
GO:0007342 & fusion of sperm to egg plasma membrane & Biological process & NOX5; SPESP1 \\
GO:0005833 & hemoglobin complex & Cellular component & HBA2; HBB \\
GO:0005721 & pericentric heterochromatin & Cellular component & IKZF1; HELLS \\
GO:0071682 & endocytic vesicle lumen & Cellular component & HBA2; HBB \\
GO:0001533 & cornified envelope & Cellular component & DSP; LCE3E \\
GO:0072562 & blood microparticle & Cellular component & CFHR3; HBA2; HBB \\
GO:0005096 & GTPase activator activity & Molecular function & TBC1D3G; TBC1D3K; TBC1D3E; TBC1D3I; TBC1D3B etc. \\
GO:0030695 & GTPase regulator activity & Molecular function & TBC1D3G; TBC1D3K; TBC1D3E; TBC1D3I; TBC1D3B etc. \\
GO:0060589 & nucleoside-triphosphatase regulator activity & Molecular function & TBC1D3G; TBC1D3K; TBC1D3E; TBC1D3I; TBC1D3B etc. \\
GO:0008047 & enzyme activator activity & Molecular function & TBC1D3G; TBC1D3K; TBC1D3E; TBC1D3I; TBC1D3B etc. \\
GO:0005344 & oxygen transporter activity & Molecular function & HBA2; HBB \\
\hline
\end{tabular}

Table 5. Results of KEGG pathway enrichment analyses on target genes.

\begin{tabular}{lccc}
\hline Term & Count & p-value & Genes \\
\hline $\begin{array}{l}\text { hsa05143: African } \\
\text { trypanosomiasis }\end{array}$ & 2 & 0.00354 & HBA2/HBB \\
hsa04145: Phagosome & 3 & 0.00671 & CD14/HLAE/TUBB2A \\
hsa05144: Malaria & 2 & 0.00686 & HBA2/HBB \\
hsa05130: Pathogenic & 2 & 0.00858 & CD14/TUBB2A \\
Escherichia coli infection & & & \\
\hline
\end{tabular}

that in HCC tissues, with a statistically significant difference $(\mathrm{p}=0.042)$.

Association between ZHX2 expression and the clinicopathological characteristics of HCC. ZHX2 expression in HCC tissues was negatively correlated with serum AFP values, with a statistically significant difference $(r=-0.246$, $\mathrm{p}=0.040$ ). ZHX2 expression was not associated with patient gender, age, tumor diameter, tumor differentiation, or serum HBsAg value ( $p>0.05$, Table 6).

Difference in PTEN expression between HCC tissues and paracancerous tissues. PTEN was positively expressed in the cytoplasm, with yellow-brown- or brown-colored staining in the cytoplasm (Figures 5C, D). The PTEN positive expression rates were $100 \%(70 / 70)$ in paracancerous tissues and 71.4\% (50/70) in HCC tissues. The rate of PTEN expression in paracancerous tissues was significantly higher than that in HCC tissues, with a statistically significant difference $(\mathrm{p}<0.001)$.

Association between PTEN expression and the clinicopathological characteristics of HCC. The expression of PTEN in HCC tissues was associated with the differentiation level of tumors, with a statistically significant difference $(\mathrm{p}=0.040)$. PTEN expression was positively correlated with the tumor differentiation $(\mathrm{r}=0.267, \mathrm{p}=0.025)$; in other words, when the tumor differentiation level was higher, the rate of PTEN expression was higher. PTEN expression was negatively correlated with the serum AFP value $(r=-0.263$, $\mathrm{p}=0.028$ ) but did not correlate with patient gender, age, tumor diameter, or serum HBsAg value ( $p>0.05$, Table 7).

Correlation analyses between ZHX2 and PTEN expression. In HCC tissues, the majority of cases with positive ZHX2 expression also had positive PTEN expression. Spearman correlation analysis revealed that the expression profiles of ZHX2 and PTEN were positively correlated in HCC tissues $(r=0.258, p=0.031)$.

\section{Discussion}

ZHX2, a member of the zinc finger and homeobox family [32], is localized on 8q13.24 and contains two zinc fingers and five homeobox domains. ZHX2 regulates various biological pathways and some disease-related genes as a transcriptional suppressor. It has been shown that ZHX2 can regulate AFP expression in HCC and bind to the eukaryotic nuclear factor NF-YA to regulate downstream gene expression $[15,33]$. This study analyzed the clinicopathological characteristics of ZHX2 in HCC and showed that ZHX2 expression negatively correlated with AFP, indicating ZHX2 could reduce AFP expression in HCC. Previous study had manifested AFP association with HCC tumor size and tumor differentiation. However, neither tumor differentiation nor tumor size was related to ZHX2 expression in this study, which demonstrated that these two clinicopathological characteristics were not only affected by AFP but other factors excluding ZHX2. This study used ChIP-seq technology to detect and investigate ZHX2-binding target genes in HCC. Association analysis between ZHX2 target genes and TSSs showed that ZHX2 binding sites were mainly localized in the intron and intergenic regions of target genes. The enrichment of ZHX2 binding sites in introns indicated that introns play a 
A

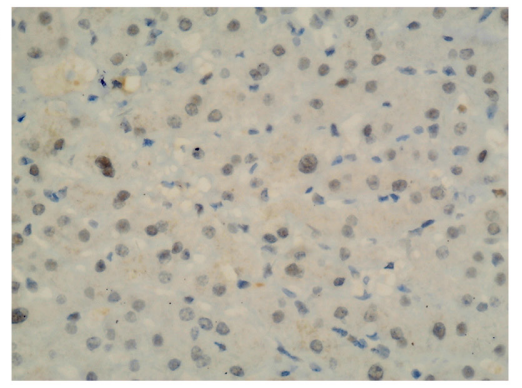

200X

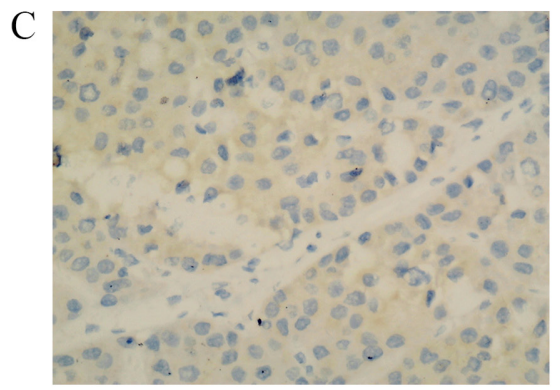

200X

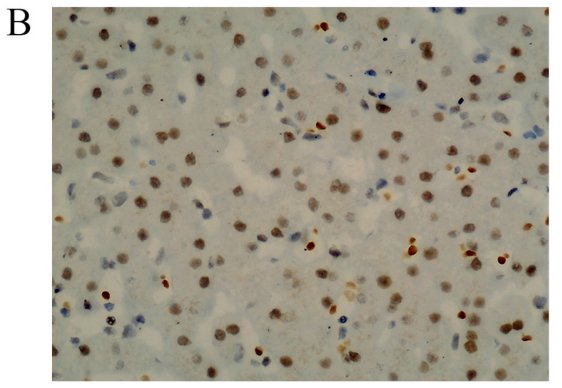

200X

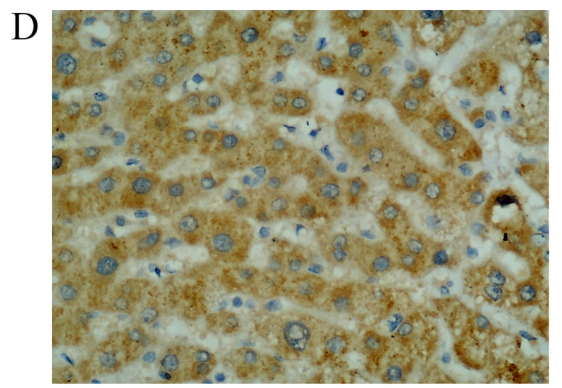

200X

Figure 5. Immunohistochemical results of ZHX2 and PTEN expression in HCC and paracancerous tissues. A) ZHX2 expression with weakly positive staining in nucleus from HCC tissues; B) ZHX2 expression with strong positive staining in nucleus from paracancerous tissues; C) PTEN expression with weakly positive staining in cytoplasm from HCC tissues; D) PTEN expression with strong positive staining in cytoplasm from paracancerous tissues.

Table 6. Associations between ZHX2 expression and clinicopathological parameters in HCC.

\begin{tabular}{|c|c|c|c|c|c|}
\hline \multirow{2}{*}{ Group } & \multirow{2}{*}{$\mathbf{n}$} & \multicolumn{2}{|c|}{ ZHX2 } & \multirow{2}{*}{$\chi^{2}$} & \multirow{2}{*}{ p-value } \\
\hline & & negative & positive & & \\
\hline \multicolumn{6}{|l|}{ Tissue } \\
\hline Cancerous & 70 & 42 & 28 & \multirow{2}{*}{4.11} & \multirow{2}{*}{0.042} \\
\hline Paracancerous & 70 & 30 & 40 & & \\
\hline \multicolumn{6}{|l|}{ Gender } \\
\hline Male & 60 & 37 & 23 & \multirow{2}{*}{ l } & \multirow{2}{*}{0.506} \\
\hline Female & 10 & 5 & 5 & & \\
\hline \multicolumn{6}{|l|}{ Age (year) } \\
\hline$<=50$ & 39 & 23 & 16 & \multirow{2}{*}{0.039} & \multirow{2}{*}{0.844} \\
\hline$>50$ & 31 & 19 & 12 & & \\
\hline \multicolumn{6}{|c|}{ Tumor diameter $(\mathrm{cm})$} \\
\hline$<=5$ & 40 & 22 & 18 & \multirow{2}{*}{0.972} & \multirow{2}{*}{0.324} \\
\hline$>5$ & 30 & 20 & 10 & & \\
\hline \multicolumn{6}{|c|}{ Differentiation level } \\
\hline High grade & 13 & 10 & 3 & \multirow{2}{*}{1.905} & \multirow{2}{*}{0.168} \\
\hline Low grade & 57 & 32 & 25 & & \\
\hline \multicolumn{6}{|l|}{ Serum HBsAg } \\
\hline Negative & 15 & 9 & 6 & \multirow{2}{*}{0.000} & \multirow{2}{*}{1.000} \\
\hline Positive & 55 & 33 & 22 & & \\
\hline \multicolumn{6}{|c|}{ Serum AFP (ng/mL) } \\
\hline$<=11$ & 32 & 15 & 17 & \multirow{2}{*}{4.231} & \multirow{2}{*}{0.040} \\
\hline$>11$ & 38 & 27 & 11 & & \\
\hline
\end{tabular}

When the theoretical frequency $<5$, Fisher's exact probability test was performed.
Table 7. Associations between PTEN expression and clinicopathological parameters in HCC.

\begin{tabular}{|c|c|c|c|c|c|}
\hline \multirow{2}{*}{ Group } & \multirow{2}{*}{$\mathbf{n}$} & \multicolumn{2}{|c|}{ PTEN } & \multirow{2}{*}{$\chi^{2}$} & \multirow{2}{*}{ p-value } \\
\hline & & negative & positive & & \\
\hline \multicolumn{6}{|l|}{ Tissue } \\
\hline Cancerous & 70 & 20 & 50 & \multirow{2}{*}{23.333} & \multirow{2}{*}{0.000} \\
\hline Paracancerous & 70 & 0 & 70 & & \\
\hline \multicolumn{6}{|l|}{ Gender } \\
\hline Male & 60 & 18 & 42 & \multirow{2}{*}{ l } & \multirow{2}{*}{0.713} \\
\hline Female & 10 & 2 & 8 & & \\
\hline \multicolumn{6}{|l|}{ Age (year) } \\
\hline$<=50$ & 39 & 12 & 27 & \multirow{2}{*}{0.208} & \multirow{2}{*}{0.648} \\
\hline$>50$ & 31 & 8 & 23 & & \\
\hline \multicolumn{6}{|c|}{ Tumor diameter $(\mathrm{cm})$} \\
\hline$<=5$ & 40 & 11 & 29 & \multirow{2}{*}{0.053} & \multirow{2}{*}{0.819} \\
\hline$>5$ & 30 & 9 & 21 & & \\
\hline \multicolumn{6}{|c|}{ Differentiation level } \\
\hline High grade & 13 & 7 & 6 & \multirow{2}{*}{ l } & \multirow{2}{*}{0.040} \\
\hline Low grade & 57 & 13 & 44 & & \\
\hline \multicolumn{6}{|l|}{ Serum HBsAg } \\
\hline Negative & 15 & 7 & 8 & \multirow{2}{*}{ I } & \multirow{2}{*}{0.109} \\
\hline Positive & 55 & 13 & 42 & & \\
\hline \multicolumn{6}{|c|}{ Serum AFP $(\mathrm{ng} / \mathrm{mL})$} \\
\hline$<=11$ & 32 & 5 & 27 & \multirow{2}{*}{4.831} & \multirow{2}{*}{0.028} \\
\hline$>11$ & 38 & 15 & 23 & & \\
\hline
\end{tabular}

When the theoretical frequency $<5$, Fisher's exact probability test was performed. 
very important role in the developmental process of HCC. However, the specific mechanisms of this relationship still require further studies. ZHX2 binding sites were mainly enriched in the intergenic regions, indicating that ZHX2 might be a transcriptional suppressor that regulates gene expression from a long distance. The long-distance regulation of target genes is more favorable for transcription factors to support their own structures to ensure the interactions among transcription factors during expression regulation. Particularly for gene regulatory steps required in the process of growth and development, these genes mainly have similar structures and are under long-distance regulation. It is generally accepted that ZHX2 regulates target genes mainly in the form of binding to the promoter regions of genes. A total of 232 genes have ZHX2 binding sites in their promoter regions, including the PTEN gene. The PTEN gene has been confirmed to be associated with HCC development and progression. PTEN is a recognized tumor-suppressor gene and can inhibit HCC development and progression. Loss of PTEN or reduction of its tumor-suppression function in HCC will cause excessive cell division of tumor cells [34]. Immunohistochemistry analysis showed that PTEN expression in HCC tissues was significantly lower than that in paracancerous tissues. In addition, with the reduction of the histopathological HCC cell differentiation level, the positive expression rate of PTEN significantly decreased. Therefore, it is hypothesized that the PTEN gene might participate in the regulation of HCC infiltration and metastasis. Inactivation of the PTEN gene influences HCC progression. The potential regulations between ZHX2 and PTEN in HCC have been demonstrated in previous studies. It was reported that ZHX2 could bind to NF-YA to repress the downstream gene including P53 [15]. And it is known that phosphorylating p53 can regulate PTEN expression in the inhibition of IGF-1/mTOR pathway. Further, the Co-IP technique confirmed that AFP could bind to PTEN and reduce the expression of PTEN, and ZHX2 could repress the expression of AFP indicating the underlying relation between PTEN and ZHX2 [35, 36]. PTEN might be a target gene regulated by ZHX2. Correlation analysis of ZHX2 and PTEN showed a positive relationship between their expression profiles, and ChIP-seq analysis revealed that the PTEN gene was bound to the ZHX2promoter region. This result confirmed that these two genes interact with each other to jointly regulate HCC development and progression. Target genes that bound to the promoter region also included $\mathrm{HBB}$, PC, MYOC, and CD14. However, the regulatory functions of these genes in HCC have been only rarely studied or reported.

GO and KEGG pathway analyses were then performed on target genes which bound in the promoter regions. GO analysis indicated that differentially expressed target genes, including PTEN, HBA2, NOX5, and CD14, were mainly associated with biological regulation, metabolic process, and management of biological processes, suggesting that ZHX2 could collaborate with transcription regulation functions to influence downstream signals to further regulate the biolog- ical behavior of HCC. In biological regulation and management of biological processes, the enriched target genes included PTEN, IKZF1, GRIN2B, HLA-E, and CD14 genes. The target genes enriched in metabolic processes were similar to the genes enriched in the above two biological processes. However, these genes and their interactive functions in HCC have been rarely studied or reported. In addition, the results also showed that target genes might be enriched in biological processes associated with tumor development and progression, including negative regulation of cell-matrix adhesion (PTEN and MYOC), adhesion connective tissues (PTEN and MYOC), and positive regulation produced by tumor necrosis factors (HLA-E and CD14). Among these genes, only PTEN has been reported to be involved in malignant tumor growth processes, such as cell proliferation in HCC. The functions of the other genes in HCC have been rarely reported, suggesting that the mechanisms underlying their interactive functions with ZHX2 in the regulation of HCC are worth of further analysis. KEGG pathway analyses indicated that these target genes were mainly enriched in the African trypanosomiasis pathway, the phagosome pathway, the malaria pathway, and the pathogenic Escherichia coli infection pathway. Most of these pathways are associated with human infectious diseases; only one pathway, the phagosome pathway, was associated with biological processes. The functions of some of the target genes involved in these pathways, such as HBA2, $\mathrm{HBB}$, and CD14, are still unknown in the context of HCC and are worth of further investigation.

This study is the first to use ChIP-seq technology to analyze transcriptionally regulated target genes of ZHX2 in HCC at the genome level. In addition to interactions with promoter regions, ZHX2 also interacted with intergenic regions to regulate target genes from long distances. Enrichment analyses of the target genes of these promoter regions revealed that the interacted target genes are associated with metabolic processes and biological regulatory processes. The pathway enrichment results suggest that the target genes might be involved in three infectious disease pathways and one phagosome pathway. One target gene, PTEN, bound to the promoter region of ZHX2, suggesting that the two genes might interact with each other and jointly regulate downstream genes to exert inhibitory functions. ZHX2 and PTEN may be involved in HCC development and progression, a finding that provides a basis for further studies of the specific regulatory mechanisms of the transcription suppressor ZHX2 in the context of HCC.

Supplementary information is available in the online version of the paper.

Acknowledgements: This study was supported by the Scientific and technological research projects in Guangxi Universities, KY2015YB065 and Guangxi Youth Scientific Foundation (No.0728057), and the Guangxi Medical University Training Program for Distinguished Young Scholars (2017). 


\section{References}

[1] CHENG S, YANG J, SHEN F, ZHOU W, WANG Y et al. Multidisciplinary management of hepatocellular carcinoma with portal vein tumor thrombus - Eastern Hepatobiliary Surgical Hospital consensus statement. Oncotarget 2016; 7: 4081640829. https://doi.org/10.18632/oncotarget.8386

[2] HUANG R, WANG X, ZHANG W, ZHANGYUAN G, JIN K et al. Down-Regulation of LncRNA DGCR5 Correlates with Poor Prognosis in Hepatocellular Carcinoma. Cell Physiol Biochem 2016; 40: 707-715. https://doi. org/10.1159/000452582

[3] HUANG W, YOU L, LIU D, YANG S, LIU M et al. S-1 plus sorafenib for the treatment of advanced hepatocellular carcinoma. J BUON 2016; 21: 1388-1393.

[4] NIU M, HONG D, MA TC, CHEN XW, HAN JH et al. Short-term and long-term efficacy of 7 targeted therapies for the treatment of advanced hepatocellular carcinoma: a network meta-analysis: Efficacy of 7 targeted therapies for AHCC. Medicine (Baltimore) 2016; 95: e5591. https://doi. org/10.1097/MD.0000000000005591

[5] QI X, ZHANG X, LI Z, HUI J, XIANG Y et al. HVPG signature: A prognostic and predictive tool in hepatocellular carcinoma. Oncotarget 2016; 7: 62789-62796. https://doi. org/10.18632/oncotarget.11558

[6] ZHANG L, JIA G, SHI B, GE G, DUAN H et al. PRSS8 is Downregulated and Suppresses Tumour Growth and Metastases in Hepatocellular Carcinoma. Cell Physiol Biochem 2016; 40: 757-769. https://doi.org/10.1159/000453136

[7] LIN J, WU L, BAI X, XIE Y, WANG A et al. Combination treatment including targeted therapy for advanced hepatocellular carcinoma. Oncotarget 2016; 7: 71036-71051. https://doi.org/10.18632/oncotarget.11954

[8] SUN JJ, CHEN GY, XIE ZT. MicroRNA-361-5p Inhibits Cancer Cell Growth by Targeting CXCR6 in Hepatocellular Carcinoma. Cell Physiol Biochem 2016; 38: 777-785. https:// doi.org/10.1159/000443033

[9] WANG F, YANG H, DENG Z, SU Y, FANG Q et al. HOX Antisense lincRNA HOXA-AS2 Promotes Tumorigenesis of Hepatocellular Carcinoma. Cell Physiol Biochem 2016; 40: 287-296. https://doi.org/10.1159/000452545

[10] WU Z, ZENG Q, CAO K, SUN Y. Exosomes: small vesicles with big roles in hepatocellular carcinoma. Oncotarget 2016; 7: 60687-60697. https://doi.org/10.18632/oncotarget.1080

[11] ZHANG Y, ZHU SL, CHEN J, LI LQ. Meta-analysis of associations of interleukin-28B polymorphisms rs8099917 and rs12979860 with development of hepatitis virus-related hepatocellular carcinoma. Onco Targets Ther 2016; 9: 32493257. https://doi.org/10.2147/OTT.S10490

[12] SHEN H, LUAN F, LIU H, GAO L, LIANG X et al. ZHX2 is a repressor of alpha-fetoprotein expression in human hepatoma cell lines. J Cell Mol Med 2008; 12: 2772-2780. https:// doi.org/10.1111/j.1582-4934.2008.00233.x

[13] MORFORD LA, DAVIS C, JIN L, DOBIERZEWSKA A, PETERSON MLet al. The oncofetal gene glypican 3 is regulated in the postnatal liver by zinc fingers and homeoboxes 2 and in the regenerating liver by alpha-fetoprotein regulator 2 . Hepatology 2007; 46: 1541-1547. https://doi.org/10.1002/hep.21825
[14] YUE X, ZHANG Z, LIANG X, GAO L, ZHANG X et al. Zinc fingers and homeoboxes 2 inhibits hepatocellular carcinoma cell proliferation and represses expression of Cyclins A and E. Gastroenterology 2012; 142: 1559-1570. https://doi. org/10.1053/j.gastro.2012.02.049

[15] MA H, YUE X, GAO L, LIANG X, YAN W et al. ZHX2 enhances the cytotoxicity of chemotherapeutic drugs in liver tumor cells by repressing MDR1 via interfering with NF-YA. Oncotarget 2015; 6: 1049-1063. https://doi.org/10.18632/ oncotarget. 2832

[16] EDMONDSON, HA; STEINER, PE. Primary carcinoma of the liver: a study of 100 cases among 48,900 necropsies. Cancer 1954; 7: 462-503.

[17] LIU HJ, GUO YY, LI DJ. Predicting novel salivary biomarkers for the detection of pancreatic cancer using biological feature-based classification. Pathol Res Pract 2017; 213: $394-$ 399. https://doi.org/10.1016/j.prp.2016.09.017

[18] FAN Q, LIU B. Identification of a RNA-Seq Based 8-Long Non-Coding RNA Signature Predicting Survival in Esophageal Cancer. Med Sci Monit 2016; 22: 5163-5172. https://doi. org/10.12659/MSM.902615

[19] LI H, HAO X, WANG H, LIU Z, HE Y et al. Circular RNA Expression Profile of Pancreatic Ductal Adenocarcinoma Revealed by Microarray. Cell Physiol Biochem 2016; 40: 1334-1344. https://doi.org/10.1159/000453186

[20] ZHUO C, LI X, ZHUANG H, TIAN S, CUI H et al. Elevated THBS2, COL1A2, and SPP1 Expression Levels as Predictors of Gastric Cancer Prognosis. Cell Physiol Biochem 2016; 40: 1316-1324. https://doi.org/10.1159/000453184

[21] ZHANG Y, HE RQ, DANG YW, ZHANG XL, WANG X et al. Comprehensive analysis of the long noncoding RNA HOXA11-AS gene interaction regulatory network in NSCLC cells. Cancer Cell Int 2016; 16: 89. https://doi.org/10.1186/ s12935-016-0366-6

[22] YANG J, WANG N. Analysis of the molecular mechanism of osteosarcoma using a bioinformatics approach. Oncol Lett 2016; 12: 3075-3080. https://doi.org/10.3892/ol.2016.5060

[23] CHEN L, LU Y, WEN J, WANG X, WU L et al. Comparative Proteomics Analysis of Mouse Habu Nephritis Models with and without Unilateral Nephrectomy. Cell Physiol Biochem 2016; 39: 1761-1776. https://doi.org/10.1159/000447876

[24] LANGKILDE A, OLSEN LC, SAETROM P, DRABLOS F, BESENBACHER $S$ et al. Pathway Analysis of Skin from Psoriasis Patients after Adalimumab Treatment Reveals New Early Events in the Anti-Inflammatory Mechanism of Anti-TNF-alpha. PLoS One 2016; 11: e0167437. https://doi. org/10.1371/journal.pone.0167437

[25] LIU Z, GUO Y, PU X, LI M. Dissecting the regulation rules of cancer-related miRNAs based on network analysis. Sci Rep 2016; 6: 34172. https://doi.org/10.1038/srep34172

[26] STANKEVICIUS V, VASAUSKAS G, BULOTIENE D, BUTKYTE S, JARMALAITE $S$ et al. Gene and miRNA expression signature of Lewis lung carcinoma LLC1 cells in extracellular matrix enriched microenvironment. BMC Cancer 2016; 16: 789. https://doi.org/10.1186/s12885-016-2825-9

[27] SU YL, HUANG HL, HUANG BS, CHEN PC, CHEN CS et al. Combination of OipA, BabA, and SabA as candidate biomarkers for predicting Helicobacter pylori-related gastric cancer. Sci Rep 2016; 6: 36442. https://doi.org/10.1038/srep36442 
[28] XU J, QIAN Y, YE M, FU Z, JIA X et al. Distinct expression profile of lncRNA in endometrial carcinoma. Oncol Rep 2016; 36: 3405-3412. https://doi.org/10.3892/or.2016.5173

[29] SONG B, DU J, FENG Y, GAO YJ, ZHAO JS. Co-expressed differentially expressed genes and long non-coding RNAs involved in the celecoxib treatment of gastric cancer: An RNA sequencing analysis. Exp Ther Med 2016; 12: 2455-2468. https://doi.org/10.3892/etm.2016.3648

[30] HU S, ZHANG M, LV Z, BI J, DONG Y et al. Expression of zinc-fingers and homeoboxes 2 in hepatocellular carcinogenesis: a tissue microarray and clinicopathological analysis. Neoplasma 2007; 54: 207-211.

[31] ZHOU X, ZHU H, LU J. PTEN and hTERT gene expression and the correlation with human hepatocellular carcinoma. Pathol Res Pract 2015; 211: 316-319. https://doi. org/10.1016/j.prp.2014.11.016

[32] KWON RJ, KIM YH, JEONG DC, HAN ME, KIM JY et al. Expression and prognostic significance of zinc fingers and homeoboxes family members in renal cell carcinoma. PLoS One 2017; 12: e0171036. https://doi.org/10.1371/journal. pone. 0171036
[33] LV Z, ZHANG M, BI J, XU F, HU S et al. Promoter hypermethylation of a novel gene, ZHX2, in hepatocellular carcinoma. Am J Clin Pathol 2006; 125: 740-746. https://doi. org/10.1309/09B4-52V7-R76K-7D6K

[34] HU TH, HUANG CC, LIN PR, CHANG HW, GER LP et al. Expression and prognostic role of tumor suppressor gene PTEN/MMAC1/TEP1 in hepatocellular carcinoma. Cancer 2003; 97: 1929-1940. https://doi.org/10.1002/cncr.11266

[35] LI M, LI H, LI C, WANG S, JIANG W et al. Alpha-fetoprotein: a new member of intracellular signal molecules in regulation of the PI3K/AKT signaling in human hepatoma cell lines. Int J Cancer 2011; 128: 524-532. https://doi.org/10.1002/ ijc. 25373

[36] PETERSON ML1, MA C, SPEAR BT. Zhx2 and Zbtb20: novel regulators of postnatal alpha-fetoprotein repression and their potential role in gene reactivation during liver cancer. Semin Cancer Biol 2011; 21:21-27. https://doi.org/10.1016/j. semcancer.2011.01.001 